\title{
Downy woodpecker foraging behavior: foraging by expectation and energy intake rate
}

\author{
Steven L. Lima* \\ Ecology, Fisheries and Wildlife Program, School of Natural Resources, University of Michigan, Ann Arbor, MI 48109, USA
}

Summary. I describe an artificial patch system that was used to study the foraging behavior of free-roaming downy woodpeckers (Picoides pubescens) in a woodlot in southeastern Michigan. The artificial "patches" used were thin logs into which were drilled small holes to hold food items (bits of sunflower seed kernels). Downy woodpeckers would systematically search the holes of a patch for food items and thus by manipulating the food distribution within the patches, the birds could be made to experience differing rates of energy intake while foraging.

Simple deterministic theories of optimal foraging in patchy environments indicate that an optimal forager, who experiences a decreasing rate of energy intake while foraging in a patch, should leave a patch when its rate of energy intake falls below the average intake rate for the overall environment. In other words, an optimal forager is continually assessing the quality of a patch and makes decisions as to when to leave a patch via its energy intake rate. When the downy woodpeckers studied could encounter any one of several types of patches each with differing, decreasing rates of energy intake, they followed a patch quality assessment strategy similar to that suggested by theory. Upon encountering a single type of patch for a number of consecutive days, however, the birds appeared to forage according to prior expectations of patch quality and not according to a quality assessment strategy based on energy intake rates. The observed expectations were not related to the number of food items per patch but they appeared to be based on expectations of when or where to leave a patch.

\section{Introduction}

Models of optimal foraging in patchy environments (see Cowie and Krebs 1979 for review) assume that foragers continually assess the quality of a patch, and make decisions as to when to leave a patch, based on their energy intake rate. One simple example of such a strategy is where a forager monitors patch-specific energy intake rates via the length of time between prey captures and leaves a patch when this time interval increases beyond some threshold (a giving-up time strategy; Krebs et al. 1974; Charnov 1976; McNair 1982).

\footnotetext{
* Present address: Department of Biology, University of Rochester, Rochester, NY 14627 U.S.A.
}

From the standpoint of optimal foraging, it is clear that an animal should not forage by expectation, i.e. decide $a$ prioi when to leave a patch and execute that decision regardless of the energy intake rate actually experienced. For example, an animal that expects to find a fixed number of prey in a patch and leaves after finding the expected number is following a number expectation strategy while a forager that expects to have to stay in a patch for a fixed length of time and leaves regardless of energy intake rate is following a time expectation strategy. Although these simple expectation foraging strategies may approximate optimal solutions to some foraging problems under special circumstances, they will, in general, lead to suboptimal behavior (Breck 1978; Iwasa et al. 1981).

Do animals foraging in patchy environments follow simple expectation strategies or are they capable of more complex patch quality assessment strategies based on energy intake rates as suggested by theory? Krebs et al. (1974), Zach and Falls (1976) and Hodges (1981) addressed this question by subjecting great tits, ovenbirds, and bumblebees, respectively, to a given patchy environment for a number of foraging trials before the environment was suddenly switched to another. Based on foraging responses to these environmental switches, each study concluded that the animals had not developed expectations but foraged according to energy intake rates in a manner suggested by Charnov's (1976) model.

Below, I describe a simple but ecologically relevant experimental system for studying the foraging behavior of free-roaming downy woodpeckers (Picoides pubescens) in patchy environments. As with previous work (Krebs et al. 1974; Zach and Falls 1976; Hodges 1981), the question of foraging by expectation vs. energy intake rate will be addressed in the framework of Charnov's (1976) model. The first set of experiments reported below will show that the woodpeckers can follow a patch quality assessment strategy that appears to be based on a giving-up-time (GUT). In the second set of experiments it will also be shown that, under some circumstances, woodpeckers may also forage according to expectations.

\section{Methods}

\section{Foraging by energy intake rate}

The first set of experiments was carried out in an oak-ashhickory woodlot in southeastern Michigan, USA, from 21 
Table 1. Patch types and their seed distributions for energy intake rate experiments. Values indicate number of seeds per level (out of a maximum of 4,0 or 1 seed/hole). A woodpecker begins foraging at level 20 and moves upward

\begin{tabular}{rllllll}
\hline \multirow{2}{*}{ Level } & \multicolumn{2}{l}{ Patch type } & & & & \\
\cline { 2 - 6 } & $\mathrm{C}$ & $\mathrm{G}$ & $\mathrm{D}$ & $\mathrm{F}$ & $\mathrm{E}$ & $\mathrm{A}$ \\
\hline 1 & 0 & 0 & 0 & 0 & 0 & 0 \\
2 & 0 & 0 & 0 & 0 & 0 & 0 \\
3 & 0 & 0 & 0 & 0 & 0 & 1 \\
4 & 0 & 0 & 0 & 0 & 0 & 0 \\
5 & 1 & 0 & 0 & 0 & 1 & 1 \\
6 & 0 & 0 & 0 & 0 & 1 & 0 \\
7 & 1 & 0 & 0 & 0 & 0 & 0 \\
8 & 1 & 1 & 0 & 1 & 2 & 1 \\
9 & 2 & 0 & 0 & 0 & 2 & 0 \\
10 & 1 & 1 & 0 & 1 & 0 & 1 \\
11 & 2 & 1 & 1 & 1 & 2 & 1 \\
12 & 3 & 1 & 0 & 0 & 3 & 1 \\
13 & 3 & 1 & 1 & 2 & 0 & 1 \\
14 & 3 & 1 & 0 & 2 & 3 & 1 \\
15 & 3 & 2 & 2 & 0 & 4 & 1 \\
16 & 4 & 1 & 0 & 2 & 0 & 1 \\
17 & 4 & 2 & 2 & 3 & 4 & 1 \\
18 & 4 & 3 & 3 & 0 & 4 & 1 \\
19 & 4 & 3 & 3 & 3 & 4 & 1 \\
20 & 4 & 3 & 3 & 3 & 4 & 1 \\
\hline
\end{tabular}

February through 1 March 1981. Patches were $95 \mathrm{~cm}$ lengths of sapling ash tress (Fraxinus spp.) $2.5-3.0 \mathrm{~cm}$ in diameter. Holes, $0.5 \mathrm{~cm}$ in diameter, were drilled $0.9 \mathrm{~cm}$ deep in groups of 4 (each hole $90^{\circ}$ from the next) at intervals of $4 \mathrm{~cm}$ (starting from the top). Twenty such groups, or levels, were present in each patch. Depending upon the patch type (see below) a given hole may or may not contain a single seed (a "seed" is approximately $1 / 20$ th of a large sunflower seed kernel (avg. wt. $=2.5 \mathrm{mg}, \mathrm{SD}=0.85$, $n=30)$ ). Each level of holes in a patch was completely covered by a strip of masking tape $1 \mathrm{~cm}$ wide and this resulted in all patches, regardless of type, being identical in appearance.

Free-roaming downy woodpeckers could be readily induced to forage on the above artificial patches and they quickly learned that there could be seeds in the tape-covered holes. Upon arriving at a patch, a bird would peck at the tape in order to locate a hole and peck through the tape to examine its contents. This is closely analogous to one of the downy woodpeckers winter foraging techniques, that of excavation (Conner 1981). A woodpecker would start foraging at the bottom level of a patch and systematically forage upward. Thus, depending on the seed distribution within a patch type (see Table 1) the birds could be made to experience differing (monotonically decreasing) rates of energy intake as they foraged upward.

The woodpeckers in the woodlot were given experience in patch use by providing them with 10 totally full $(1 \mathrm{seed} /$ hole) patches each morning for the 6 days prior to the start of experimentation on day 1 and on each of these days, each hole in every patch was opened by the birds. For the experimental period (days 1-8), foraging trials were allowed to begin at dawn and only 4 patches were made available (hung $3 \mathrm{~m}$ apart from trees) to the birds at any one time. There was no specific order of patch type presen-
Table 2. Patch types and their seed distributions for expectation experiments. Values in table indicate number of seeds per level (out of a maximum of 4,0 or 1 seed per hole). A woodpecker usually begins foraging at level 10 and moves upward

\begin{tabular}{llll}
\hline Level & \multicolumn{2}{l}{ Patch type } & HD \\
\cline { 2 - 4 } & F & HF & 1 \\
\hline 1 & 3 & 0 & 2 \\
2 & 3 & 0 & 1 \\
3 & 3 & 0 & 2 \\
4 & 3 & 0 & 1 \\
5 & 3 & 0 & 2 \\
6 & 3 & 3 & 1 \\
7 & 3 & 3 & 2 \\
8 & 3 & 3 & 1 \\
9 & 3 & 3 & 2 \\
10 & 3 & 3 & \\
\hline
\end{tabular}

tation. At the end of each morning's foraging trials, all unused patches (5-10, of various types) were left out for the birds to use at a later time. Thus, the birds had more experience with the patches than the data below indicate.

Usually, all 4 patches available to a bird were visited during a foraging bout and they were immediately collected and replaced with fresh patches for another bird to use. For each individual patch visit, using stopwatches, the time at which a bird left one level to go on to the next was recorded as was the total time a woodpecker foraged on the patch. Towards the top of a patch, an effort was made to monitor individual seed captures in order to estimate the GUT. Each of these visited patches was later analyzed to determine how many holes were opened and how many seeds were found at each level. From this and the time data specific to each visited patch, average cumulative seed intake curves could be constructed for each patch type. Only the results from the 3 patch types for which I have the most data $(C, D$ and $G)$ will be presented in detail (Fig. 3) although a total of 6 patch types were used (Table 1).

The woodpeckers were never seen to "rest" or exhibit any non-foraging behavior while on a patch. Occasionally, however, a woodpecker just arriving at the study site would displace another from a patch or a woodpecker would abruptly leave a patch and the study area. These data are excluded from the analysis. As the birds could not reliably be distinguished, all the data were pooled for analysis. There were, however, at least 3 individual woodpeckers (all males) participating.

\section{Foraging by expectation}

The second set of experiments was carried out at a different site in the same woodlot described above ( 9 months later). The artificial "patches" used were similar to those described above with the exception that they had only 10 levels of holes/patch instead of 20 . Smaller patches (with less food) were used to increase the number of patches visited per day. Three patch types were used: full (F), halffull (HF), and half density (HD) (see Table 2 for distribution of seeds in patch types).

The woodpeckers were given experience in patch use with $F$ patches for 12 days prior to the start of experimenta- 
tion (12 November 1981, Day 1). On experiment days 1-5, only $F$ patches were available to the woodpeckers. On days 6-16, HF patches were available and for day 17 only, HD patches were available. $F$ and HF patches were again used during days 18-21 and days 22-24, respectively (Fig. 4). At least 25 patches, hung $3 \mathrm{~m}$ off the ground and 4-6 m apart, were available to the birds at any time during the day. Only one patch type was present over a given period of days.

On each experimental day, a half hour before dawn, exploited patches from the previous day were removed and replaced with fresh ones. The number of holes opened and seeds found per level were recorded for each day's exploited patches. On some days, I would remain at the study site to observe patch visitation. The 2 downy woodpeckers in the study area, apparently a mated pair, were the only birds seen visiting the patches. If patch visitation was observed, the total time spent on each patch as well as the sex of the foraging bird was recorded.

\section{Results}

\section{Foraging by energy intake rate}

Figure 1 shows the average GUTs for each day of the study. The average GUT was not constant over time (one-way ANOVA, $P<0.001$ ). On days 1 and 2 , the GUT averaged about $45 \mathrm{~s}$ while after day 2 , the GUT appeared to have "stabilized" (ANOVA, $P>0.20$ ) at an average of $20.35 \mathrm{~s}$ $(n=54, \mathrm{SE}=0.80)$. Also, for days $3-8$, the average GUTs according to patch type (Fig. 2) were not significantly different (ANOVA, $P>0.20$ ). As Charnov's (1976) theory would suggest, there was no significant correlation between (for days 3-8) GUT and total foraging time ( $\mathrm{r}=0.086, n=$ $54, P>0.30$ ), GUT versus total number of holes opened $(\mathrm{r}=0.21, n=54, P>0.15)$ or GUT versus total seeds found $(\mathrm{r}=0.0054, n=54, P>0.20)$.

In Fig. 3, the average total seeds acquired versus time spent foraging (seed accumulation functions) on patch types $C, D$ and $G$ are shown. Only patches visited during the period of "stable" behavior, days 3-8, were considered. The woodpeckers foraged significantly longer on patch type $\mathrm{C}$ than on $\mathrm{D}$ or $\mathrm{G}$ (oneway ANOVA, $P<0.05$ ). Although the birds foraged longer on $G$ than $D$, the difference was not significant ( $t$-test, $P>0.05$ ). The woodpeckers, however, appeared to forage longer on the more profitable patch types.

As Charnov's (1976) key assumption (monotonically decreasing energy intake rate) was met, the derivatives of the seed accumulation functions at the observed average total foraging times (Fig. 3), should be equal for each patch type and be the average rate of energy intake for the overall environment. For patches $D$ and $G$, the observed derivative values were almost equal $(D, 0.051$ seeds/s; $G, 0.052$ seeds/ s) while that for patch $C$ was somewhat higher $(0.061$ seeds/ s). As these values were taken directly from fitted curves, there was no direct way of testing for significance between them. A crude comparison was obtained by evaluating the seed accumulation function derivatives at the the endpoints of the $95 \%$ confidence intervals of the average total foraging time for each patch type and then comparing the overlap of the resulting ranges. All 3 ranges overlap greatly (C [0.0483-0.0769]; G [0.0380 0.0677], D [0.0379-0.0654]), so I assume that there were no significant differences.

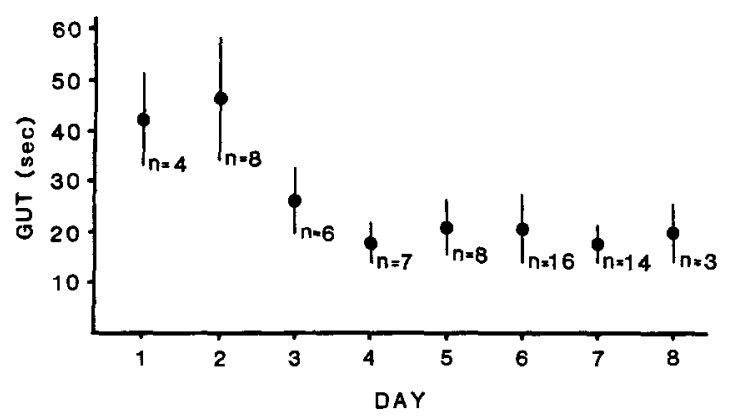

Fig. 1. Giving-up-times for days 1-8 (average $\pm 1 \mathrm{SD}$, all patch types included)

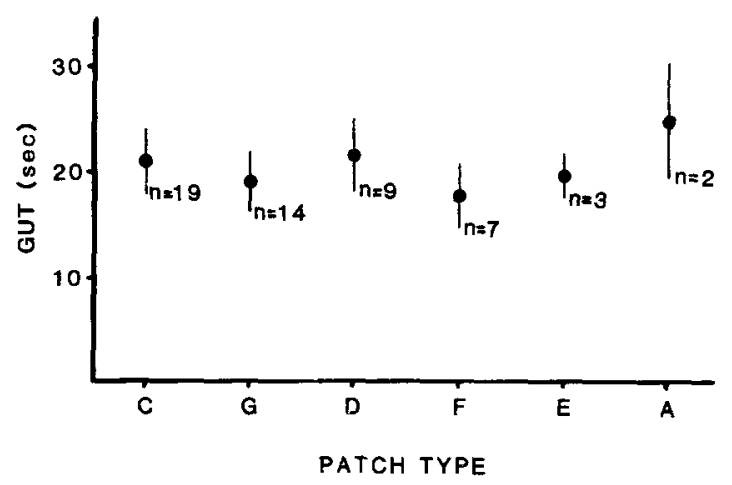

Fig. 2. Giving-up-times for each patch type (average $\pm 1 \mathrm{SD}$ ) for days 3-8 only (see text)

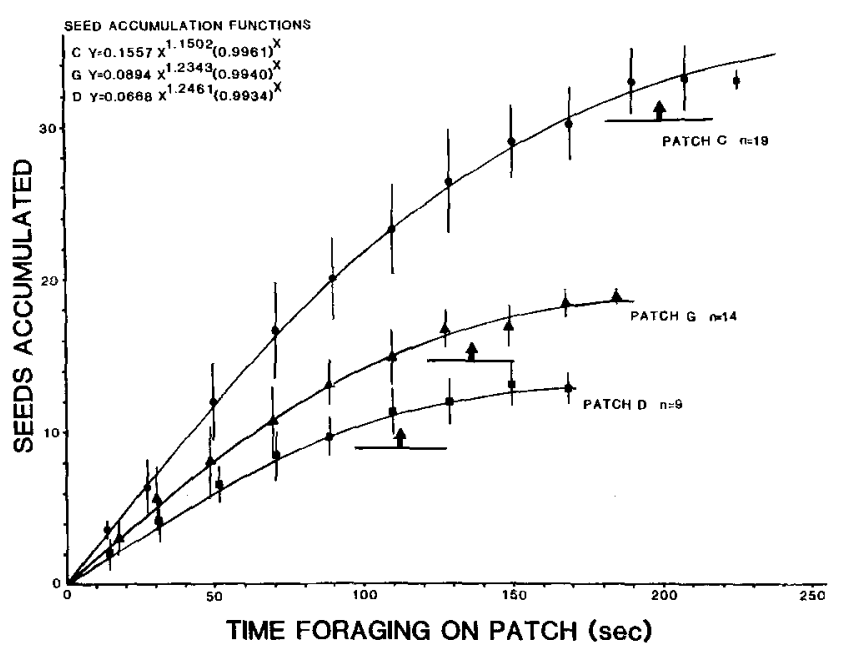

Fig. 3. Average cumulative seed intake for patch types $C, D$, and G. Points on curves represent average accumulated seeds for $20 \mathrm{~s}$ intervals ( \pm 1SD). The equation (seed accumulation function) for each fitted line is given $\_$indicates average total foraging time on a patch type and its $95 \%$ confidence interval

Based on the results from this first set of experiments, it appeared, from the lag in the GUT response (Fig. 1), that the woodpeckers had initially formed some sort of expectation with regard to the totally full patches that they were provided with prior to the start of experimentation on day 1 (Cowie and Krebs 1979). The following results are from experiments aimed to clarify the nature of this expectation. 


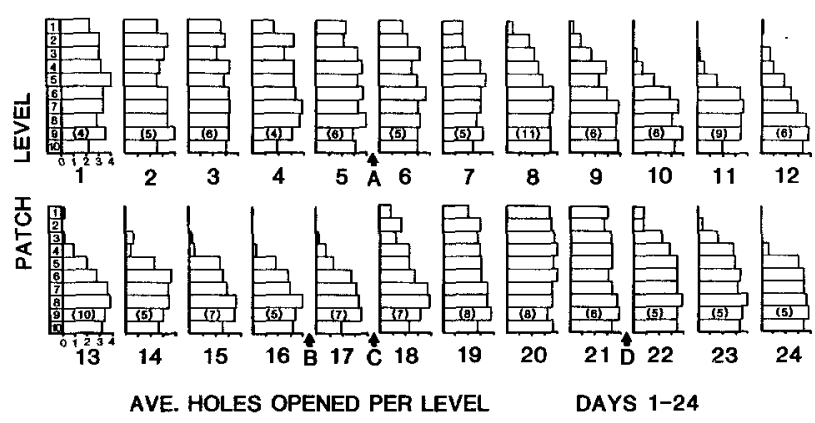

Fig. 4. Patch hole opening patterns for days 1-24 of expectation experiments. Each graph represents the hole opening pattern for the average patch of that day. The maximum possible number of holes opened per level is 4 . A, B, C and D denote patch type switches: $A: F$ to $\mathrm{HF}, \mathrm{B}: \mathrm{HF}$ to $\mathrm{HD}, \mathrm{C}: \mathrm{HD}$ to $\mathrm{F}, \mathrm{D}: \mathrm{F}$ to HF. Numbers in parentheses indicate number of patches visited on a given day

\section{Foraging by expectation}

For this set of experiments, unlike in the first set, patches were available to the birds throughout the day. As in the first set of experiments, woodpeckers would arrive at the study site just after dawn to forage. Occasionally, some patches were visited just before nightfall and from trips made to the study site during the day I found that patches were rarely visited at other times. Out of the 150 recorded visits, 31 , evenly dispersed throughout the experimental period, were actually observed. Of these visits $32 \%$ were made by the female and $68 \%$ by the male. The average foraging time per hole opened was $4.2 \mathrm{~s}(\mathrm{SD}=0.91)$ with no significant difference noted between the birds (t-test, $P>0.05$ ). For the data analysis all observations were combined.

Hole opening patterns. The average number of holes opened per level (out of a maximum of 4) for patches visited during each day of the second set of experiments is shown in Fig. 4. Although the data may be analyzed in detail according to individual patch levels, total holes opened per patch, etc., only the major results will be presented as most points are obvious from Fig. 4.

1. Days 1-5, F patches: The patch hole-opening pattern for days $1-5$ was relatively stable with $3-4$ holes opened per level, i.e., the woodpeckers were foraging all the way up the patches. However, there were significantly fewer holes opened at level 10 (Median test, $P<0.05$ ). This is due to the fact that the woodpeckers sometimes started foraging at level 9 instead of 10 .

2. Days 6-16, HF patches: For days 6 and 7, the holeopening patterns appeared similar to days $1-5$ and no significant differences were indicated within or between levels (Median tests, $P>0.05$ ). On day 8 , significantly fewer holes were opened at level 1 (Median test, $P<0.05$ ). Through days $8-10$, the woodpeckers gradually started to leave the patches earlier. For days $10-16$, there were no significant differences within or between levels (Median tests, $P>0.05$ ) and the hole opening pattern appeared to have stabilized with an average of $3.14(\mathrm{SD}=3.17)$ holes opened in the empty region of the HF patches (levels 1-5, Table 2).

3. Day 17, HD patches: From Fig. 4 it appears that the hole-opening pattern on day 17 was not different from those of days 10-16. Statistical analysis within and between levels shows this to be the case (Median tests, $P>0.05$ ).
4. Days 18-21, F patches: It is clear that the woodpeckers foraged farther up the patches immediately following the HD to F switch on day 18 (switch C, Fig. 4). The hole-opening pattern quickly stabilized to where almost all the holes were opened for all patches visited during days 19-21.

5. Days 22-24, HF patches: Immediately after the switch from $F$ to $H F$ patches (switch D), significantly fewer holes were opened at levels 1 and 2 only (Median tests, $P<0.05$ ). By day 24 , few holes were being opened in the empty region of the HF patches.

\section{Discussion}

\section{Foraging by energy intake rate}

This study does not attempt to independently ascertain the average energy intake rate for the environment, so the optimal GUT and thus the optimal total foraging time for each patch type could not be calculated a priori. Thus, as Krebs (1978) points out, such studies are not rigorous tests of Charnov's (1976) theory. These experiments, however, were undertaken to demonstrate that the woodpeckers can forage according to energy intake rates. In any case, some predictions follow from Charnov's (1976) model and these can be tested.

1. The woodpeckers should forage longer on the more profitable patch types.

2. Each patch type should be left at the same threshold rate of energy intake.

3. GUTs should be relatively constant over the short run.

For days 3-8, all of the above predictions were substantiated. However, if we consider days $1-8$, they do not hold. On the first two days of experimentation, the average GUT was twice that of the "stabilized" GUT (days 3-8). One way to explain this would be that the woodlot environment suddenly improved and therefore the optimal GUT decreased. This is highly unlikely in late winter. A more likely explanation is that the response "lag" represented the formation of an expectation by the woodpeckers. Prior to day 1 , the woodpeckers, had experienced only totally full patches and it appears that they expected these full patches on day 1 and were not closely monitoring energy intake rate as theory assumes (see below).

\section{Foraging by expectation}

As mentioned earlier, other studies of foraging behavior in patchy environments (Krebs et al. 1974; Zach and Falls 1976; Hodges 1981) did not indicate foraging by expectation, although Krebs et al. (1974) and Zach and Falls (1976) could not convincingly rule out time expectations. In the present study, evidence for expectations was investigated with the same approach used in the above studies: downy woodpeckers were subjected to a given "environment" which was then suddenly switched to another. A total of 4 switches were made over the 24-day period and each will be considered separately.

1. Switch A: Prior to switch A (days 1-5), the woodpeckers had experienced only full $(F)$ patches and they generally opened 3-4 holes/level throughout the patches they visited.

Prediction: If woodpeckers were "expecting" full 
patches on day 6 (first day after switch to HF patches, Fig. 4), they would open significantly more holes on day 6 than on subsequent days (of HF patches).

Outcome: Upon switching from the $F$ to the HF patches on day 6 , no significant differences from the hole opening patterns observed on days 1-5 were observed until day 8 (Fig. 4), even though no seeds were being found above level 6 (Table 2). From these results, it appears that the prediction is upheld; the woodpeckers expected $F$ patches on day 6 and executed that expectation, regardless of energy intake.

2. Switch B: Although switch A indicated foraging by expectation, a position expectation (an expectation of where to leave the patch), a time expectation (an expectation of how long to remain on a patch) or a number expectation (forage until the 30 seeds in the $F$ patches were found) can all explain the observed behavior equally well. Based on the relatively stable behavior observed during days 10-16, it was assumed that another expectation of the same type as indicated by switch $\mathrm{A}$ had reformed regarding the HF patches. To distinguish a number expectation from the other two expectations, the patches were switched from HF to HD on day 17. HD patches have the same number of seeds (15) as HF patches, but they are spread out over levels 1-10 (Table 2).

Prediction: If the woodpeckers had a number expectation of receiving about 15 seeds/patch during days 10-16, they would have foraged all the way up the HD patches on day 17 to achieve the same total seed intake as on days 10-16. If they had a time or position expectation regarding the HF patches, their behavior on day 17 would not differ from days 10-16.

Outcome: It is clear that an expectation other than a number expectation had formed. For days 10-16, the woodpeckers found an average of 12.1 seeds/patch $(\mathrm{SD}=2.3)$. For day 17 , an average of only 5.1 seeds/patch were found before leaving to visit another patch and the hole opening pattern for day 17 is not different from days 10-16 (Fig. 4).

3. Switch C: Switch B above demonstrated the formation of an expectation for HF patches. On day $18, F$ patches were again made available.

Prediction: If the woodpeckers were foraging according to a time or position expectation during days 10-17, they should have left the F patches at level 5 or 6 on day 18 as they did on days $10-17$.

Outcome: The results show that this prediction is not strictly upheld. Out of the 7 recorded visits on day 18 , only 2 conform to the prediction while on the other 5 visits the birds exploited the patches through levels 1 or 2. During days 19-21, total patch exploitation was the rule (Fig. 4). Apparently, for those patch visits on day 18 where the woodpeckers proceeded past level 5 , they continued on as more and more seeds were found. Thus, to some extent, the birds were monitoring their intake rate. The behavior of opening 3-4 holes above level 6 during days 10-16 may be regarded as sampling behavior.

4. Switch D: By day 21 , the birds had experienced all 3 patch types. After 4 days (18-21) of F patches, the patches were switched to determine if the birds had again formed an expectation or somehow would "keep in mind" that encountering other patch types was possible.

The switch from $F$ to HF patches on day 22 indicates an expectation had reformed. But none of the patches visited on day 22 were fully opened. By day 24 , the hole opening pattern was not significantly different from days
10-16 (both within and between levels, Median tests, $P>$ 0.05 ). Thus, compared to switch $\mathrm{A}$, the adjustment to $\mathrm{HF}$ patches after switch $D$ was rapid.

From evidence presented by Cowie and Krebs (1979), it appears that great tits may be using expectations while foraging in patchy laboratory environments. Cowie and Krebs found evidence of time expectations during switches from poor to good as well as from good to poor environments. In the present study, an expectation was clearly demonstrated only for the "good" to "poor" switches (switches A, B and D). Switch C (poor to good) was detected relatively quickly by the birds. This is most likely due to the fact that any sampling behavior in the empty region of the HF patches (above level 6) exhibited during days 10-16 was immediately rewarded on the F patches of day 18 and the woodpeckers responded accordingly. It is unlikely that such immediate rewards followed any sampling behavior by the tits studied by Cowie and Krebs (1979). It should be pointed out that the data in this study cannot distinguish between a position or a time expectation, nor can it be determined if the two operate together.

Patch visitation rates during the second set of experiments were quite low (Fig. 4) and it is curious that patches were visited only at dawn and sometimes at dusk even though patches were available all day. One would expect that energy-maximizing woodpeckers would make more visits, throughout the day. Instead, the birds spent the great majority of their time foraging elsewhere in the woodlot. Perhaps the seeds used were not very palatable or perhaps the food is of low quality and diet diversification was called for. From other experiments with this woodpecker-foraging system, however, it has been found that patch visitation rates are much higher in the late winter (Lima, in press). It seems that with the colder temperatures and potential problems with food depletion experienced during the late winter, the birds are far more willing to visit patches and will do so at any time of the day.

\section{Conclusions}

When faced with an unchanging environment with patches of constant profitability, downy woodpeckers will forage according to (flexible) expectations. It is also clear, however, that the woodpeckers can adopt foraging strategies where "on patch" quality assessment is called for. After the start of the first set of experiments, the woodpeckers could encounter any one of 6 patch types, each being superficially identical. In this more variable environment, once the initial expectation had been overcome, woodpeckers followed a patch assessment strategy consistent with Charnov's (1976) model and a special case of McNair's (1982) model (all patches identical in appearance).

Although the downy woodpeckers foraged, generally, as predicted, the major assumption underlying the deterministic model employed (continuous energy intake monotonically, smoothly decreasing with time spent on the patch) is likely to be violated in the natural patches encountered by woodpeckers. Oaten's (1977) stochastic model of patch foraging, which does not require the restrictive conditions of Charnov's (1976) model, is more likely to be appropriate to the real world foraging problems faced by the woodpeckers. It should be noted that discrete stochastic versions of Charnov's model have proven useful (Pyke 1981; Zimmerman 1981; and Hodges 1981). Using free-roaming 
downy woodpeckers and the artificial patch system, I have also conducted experiments designed to test a more realistic, stochastic foraging model (Lima, in press). The results show that downy woodpeckers are capable of much more complex foraging strategies than simple expectations or GUTs, although foraging expectations similar to those described above were also observed in these stochastic experiments.

Acknowledgements. This manuscript was improved considerably by the comments of G. Belovsky, J.S. Belovsky, G. Bourne, D. Schluter and anonymous reviewers. I thank A. Ferguson for typing the manuscript and her patience.

\section{References}

Breck JE (1978) Suboptimal foraging strategies for a patchy environment. Ph.D. dissertation, Michigan State University

Charnov EL (1976) Optimal foraging, the marginal value theorem. Theor Pop Biol 9:129-136

Conner RN (1981) Seasonal changes in woodpecker foraging patterns. Auk 98:562-570

Cowie RJ (1977) Optimal foraging in great tits (Parus major). Nature 268:137-139

Cowie RJ, Krebs JR (1979) Optimal foraging in patchy environments. In: RM Anderson et al. (eds), Population dynamics. Oxford Blackwell, p 183-206
Hodges CM (1981) Optimal foraging in bumblebees: hunting by expectation. Anim Behav 29:1166-1171

Iwasa Y, Masahiko H, Yamamura N (1981) Prey distribution as a factor determining the choice of optimal foraging strategy. Am Nat 17:710-723

Krebs JR (1978) Optimal foraging: decision rules for predators. In: Krebs JR, Davies NB (eds), Behavioral Ecology. Sinauer Associates Sunderland, Massachusetts

Krebs JR, Ryan JC, Charnov EL (1974) Hunting by expectation or optimal foraging? A study of patch use by chickadees. Anim Behav 22:953-964

Lima SL (in press) Downy woodpecker foraging behavior: efficient sampling in simple stochastic environments. Ecology

McNair JN (1982) Optimal giving-up times and the marginal value theorem. Am Nat 119:511-529

Oaten A (1977) Optimal foraging in patches: a case for stochasticity. Theor Pop Biol 12:263-285

Pyke GH (1978) Optimal foraging in hummingbirds: testing the marginal value theorem. Am Zool 18:739-752

Pyke GH (1981) Honeyeater foraging: a test of optimal foraging theory. Anim Behav 29:878-888

Zach R, Falls BJ (1976) Do ovenbirds (Aves:Parulidae) hunt by expectation? Can J Zool 54:1894-1903

Zimmerman M (1981) Optimal foraging, plant density and the marginal value theorem. Oecologia (Berlin) 49:148-153

Received August 11, 1982 